\title{
Optimization of Radar Scanning Strategies Using an Ensemble Relative Error Criterion
}

\author{
Luther White \\ Department of Mathematics \\ University of Oklahoma \\ Norman, Oklahoma 73019 \\ lwhite@ou.edu \\ and \\ Alan Shapiro \\ School of Meteorology \\ University of Oklahoma \\ Norman,Oklahoma 73019 \\ ashapiro@ou.edu
}

May 25, 2006

\begin{abstract}
We study the formulation of the problem to retrieve wind fields from radar data. The considerations here view the radar locations as fixed with retrieved wind fields as functions of scanning strategies. The scanning strategies are formulated in terms of a scanning model that depends on certain parameters. The objective here is to minimize a functional that is defined in terms of the relative error between retrieved wind velocities and an ensemble of test wind fields. A numerical study is presented illustrating the theory developed.
\end{abstract}

\section{Introduction.}

The wind field retrieval problem seeks to estimate a three dimensional wind field within a domain $\Omega$ from radar data consisting of measurements of radial velocities from $n$ radar sites. Admissible wind fields belong to a suitable Hilbert space consisting of functions from $\Omega$ into $\Re^{3}$. The retrieval problem is formulated as a minimization problem on the Hilbert space of admissible vector fields under weakly constrained physical laws and regularization. The existence of a unique solution to this problem is then a consequence of classical Hilbert space theory. The problem, however, depends on parameters describing the radar measurement model that must be specified in its formulation. The radar measurement 
model consists of the description of the location of the radar, the radar beam geometry, the observational region for the radar, and the scanning strategy. The solution of the retrieval problem depends on these parameters, and thus, they may be considered as controls around which optimization problems may be designed. In this study the location of the radars is considered fixed as well as sweep angles, that is the scanning sector angles, of the radar. The parameters of interest are described in terms of an $n$-tuple of scanning directions vectors where the radars are pointed. Our objective here is to consider the determination of these parameters minimizing a general retrieval error defined over ensembles of possible winds that capture uncertainty in estimates as the wind fields evolve as functions of time.

The synthesis of three-dimensional vector wind wind fields from Doppler radar data is an important part of mesoscale research and operational meteorology, with particularly vital applications in hazard warning and nowcasting (e.g., tornado detection and prediction), and in numerical weather prediction. Techniques of single-Doppler velocity retrieval vary in complexity from the simple Velocity Azimuth Display (VAD), in which the imposed model is a wind field that varies linearly with the spatial coordinates, to the full model adjoint techniques in which the radial wind obtained from time integration of the complete dynamical equation set of a numerical weather prediction model is fit to radial wind observations over a window of time. Dual-Doppler wind retrieval techniques may also be couched in an adjoint or other variational framework. Key developments in the history of single- and multiple-Doppler wind retrievals, and some of the remaining problems are summarized in Shapiro et al [11].

The considerations here are related to work in the meteorological community on adaptive measurements, see [1] and references therein. There, measurement procedures are determined to optimize certain criteria. The Kalman filter (actually the ensemble transform Kalman filter) is used to capture the evolution of predictions. Operators within the filter depend on parameters that specify the measurement process. Selection of parameters is base on criteria such as minimization of the estimate error variance. In this work we directly consider the minimization of the estimation relative error. In subsequent work we consider criteria based on ensemble Kalman filtering and ensemble transform Kalman filters applied to radar scanning problems.

In Section 2 the general Hilbert space formulation that was originally presented in [14] is given in sufficient detail for application here. The retrieved wind velocity is obtained as the solution of a variational boundary value problem that depend on scanning parameters. Properties of the retrieved velocity as a function of the scanning parameters are examined, and it is shown that the retrieved wind fields are differentiable with respect to the scanning directions. In Section 3 an optimization procedure is formulated. As an event of interest evolves in time, a scanning strategy is determined in terms of parameters minimizing a relative error functional over an ensemble of possible perturbations to 
the current estimated wind field advancing the wind field to the next time instant. The scanning parameter that optimizes relative error over the generated ensemble is then used to collect data in the next step to obtain a new estimate of the wind field. In Section 4, results of a numerical study are discussed for an example with two radar tracking wind fields resulting from a moving vortex. In this simple case the set of admissible scanning parameters consists of four directions for each radar.

\section{Retrieval of Wind Fields from Radar Data.}

The retrieval of wind fields from radar data is posed as a minimization problem seeking wind fields matching data under various constraining models. To pose the problem of estimating wind field information from radar data requires the specification of a retrieval functional that includes terms involving a data model (radar measurement model), a physics-based model, and a regularization. The model describing the relation between observed radar data and the vector-valued function constituting the actual wind field defines a mapping whose output is the radar data associated with that wind field. This mapping depends on the specifics of the measurement process. The physics-based model is used to constrain the wind field. It aids in the interpolation between radar sites in a physically reasonable way. Finally, a regularization term is included in the retrieval functional to assure that the associated minimization problem has a unique solution.

To formulate the retrieval problem, let $\Omega$ denote an observational volume that, for ease, is a rectangular volume of points $\mathbf{x}=(x, y, z)^{T}$ in $\Re^{3}$ such that

$$
\Omega=\left\{\mathbf{x}: 0<x<L_{x}, 0<y<L_{y}, 0<z<L_{z}\right\}
$$

and with its base denoted by

$$
\Omega_{0}=\left\{(x, y, 0)^{T}: 0<x<L_{x}, 0<y<L_{y}\right\} .
$$

Assume there are $n$ radar site locations $\mathbf{x}_{1}, \ldots, \mathbf{x}_{n}$ in $\Omega_{0}$. Define the vector-valued functions from $\Omega$ into $\Re^{3}$ that are used to describe the wind field within $\Omega$.

$$
\begin{aligned}
& \mathbf{v}_{s}(\mathbf{x})=\text { velocity of scattering particles in the sample volume } \Omega \\
& \qquad \mathbf{v}(\mathbf{x})=\text { air velocity }: \mathbf{v}(\mathbf{x})=v_{1}(\mathbf{x}) \mathbf{i}+v_{2}(\mathbf{x}) \mathbf{j}+v_{3}(\mathbf{x}) \mathbf{k}
\end{aligned}
$$

with $\mathbf{v}=\left(v_{1}, v_{2}, v_{3}\right)^{T}$ a column vector unless otherwise indicated. Let

$$
\mathbf{v}_{t}(\mathbf{x})=\text { terminal velocity of the scatterers : } \mathbf{v}_{t}(\mathbf{x})=W_{t}(\mathbf{x}) \mathbf{k}
$$

where $W_{t}<0$

$$
\mathbf{v}_{s}(\mathbf{x})=\mathbf{v}(\mathbf{x})+\mathbf{v}_{t}(\mathbf{x})
$$


Define the vector-valued function emanating from the ith radar location $\mathbf{x}_{i}$ and pointing toward the point $\mathbf{x} \in \Omega$

$$
\mathbf{r}_{i}(\mathbf{x})=\mathbf{r}\left(\mathbf{x}, \mathbf{x}_{i}\right)=\frac{\mathbf{x}-\mathbf{x}_{i}}{\left|\mathbf{x}-\mathbf{x}_{i}\right|} \text { for } \mathbf{x} \neq \mathbf{x}_{i} \text { and } \mathbf{0} \text { for } \mathbf{x}=\mathbf{x}_{i}
$$

The radial velocity observed at the ith radar is then expressed in terms of the dot product

$$
v_{r}\left(\mathbf{x}, \mathbf{x}_{i}\right)=\mathbf{r}_{i}(\mathbf{x}) \cdot \mathbf{v}_{s}
$$

The real-valued function

$$
\mathbf{x} \mapsto v_{r}\left(\mathbf{x}, \mathbf{x}_{i}\right)
$$

defined on $\Omega$ expresses the radial velocity corresponding to that observed at the point $\mathbf{x}$ (assuming the point is within the coverage set associated with the ith radar) from a radar located at the point $\mathbf{x}_{i}$. In this case the function $v_{r}\left(\mathbf{x}, \mathbf{x}_{i}\right)$ is to be compared with an observation $v_{r i}(\mathbf{x})$.

To model the coverage of the ith radar, we define a function from $\Omega$ into $\Re$

$$
\mathrm{x} \mapsto \phi_{i}(\mathbf{x})
$$

taking the value 1 over the coverage set of the ith radar and the value 0 in the complement of the coverage set. The coverage set is determined as a sector centered on a direction indicated by the unit vector

$$
\mu(\alpha)=[\cos (\alpha) \sin (\alpha) 0]^{T}
$$

where $\alpha \in[-\pi, \pi)$.

Remark 2.1. For the purposes of this work, a coverage function is defined for each radar location $\mathbf{x}_{i}$ for $i=1, \ldots, n$ in terms of a characteristic function defined over a cylindrical set with a cross section consisting of a conical sector $C$ centered on the vector $\mathbf{u}\left(\alpha_{i}\right)$, of angular measure $\beta$, and of radius $R$

$$
\Xi_{C}(\mathbf{x})=1 \text { if } \mathbf{x} \in C \text { and }=0 \text { otherwise. }
$$

Thus, of interest for a radar located at $\mathbf{x}_{i}$ are sets

$$
C_{i}(\alpha)=\left\{\mathbf{x}:\left|\mathbf{x}-\mathbf{x}_{i}\right| \leq R, \mathbf{r}\left(\mathbf{x}, \mathbf{x}_{i}\right) \cdot \mu(\alpha) \geq \gamma=\cos (\beta)\right\}
$$

so that

$$
\phi_{i}(\mathbf{x})=\phi_{i}(\mathbf{x}, \alpha)=\Xi_{C_{i}(\alpha)}(\mathbf{x})
$$

The set $C_{i}(\alpha)$ is the scanning set for the radar located at the point $\mathbf{x}_{i}$ in the direction of $\mu(\alpha)$. 
Remark 2.2. In the present work we consider the coverage functions as defined above. The locations $\mathbf{x}_{i}, i=1, \ldots n$ are fixed and the admissible set are the coverage functions determined by the direction unit vectors $\mathbf{u}\left(\alpha_{i}\right)$ for $\alpha_{i} \in[-\pi, \pi)$.

Remark 2.3. The observation from the radar at site $\mathbf{x}_{i}$ is given by $v_{r}\left(\mathbf{x}, \mathbf{x}_{i}\right)$ for those $\mathbf{x}$ in $C_{i}(\alpha)$. The resulting radial velocities from simulated wind fields are compared through the observation with $v_{r_{i}}$ in $C_{i}(\alpha)$ by integrating their differences over the set $C_{i}(\alpha)$. It is useful to introduce an observation as $\phi_{i}(\mathbf{x}, \alpha) v_{r_{i}}(\mathbf{x})$.

The second term included in the retrieval functional is associated with the underlying physics-based model. In this work, the anelastic continuity equation is introduced to constrain wind fields, cf. $[6,9,10,14]$

$$
\nabla \cdot(\rho \mathbf{v})=0
$$

where $\rho$ is the density. Because the applications considered in this work involve relatively small altitudes, the density $\rho$ is taken to be constant. Hence, we use the divergence free condition

$$
\nabla \cdot \mathbf{v}=0
$$

Finally, terms are included to assure that the retrieval problem has a unique solution. Hence, a term equivalent to

$$
\int_{\Omega}\left\{\left|\nabla v_{1}\right|^{2}+\left|\nabla v_{2}\right|^{2}+\left|\nabla v_{3}\right|^{2}+|\mathbf{v}|^{2}\right\} d \mathbf{x}
$$

is included.

To pose the problem in a function space setting, let $H=L^{2}(\Omega)$ and $V=$ $H^{1}(\Omega)$. Also, we introduce the Hilbert space

$$
\mathbf{H}=L^{2}\left(\Omega, \Re^{3}\right)
$$

with the inner product

$$
(\mathbf{u}, \mathbf{v})=\int_{\Omega} \mathbf{u}^{T} \mathbf{v} d \mathbf{x}
$$

and norm

$$
\|\mathbf{u}\|_{H}=(\mathbf{u}, \mathbf{u})^{\frac{1}{2}}
$$

along with the space

$$
\mathbf{V}=H^{1}\left(\Omega, \Re^{3}\right)
$$

with inner product

$$
(\mathbf{u}, \mathbf{v})_{V}=\int_{\Omega}\left\{\nabla u_{1} \cdot \nabla v_{1}+\nabla u_{2} \cdot \nabla v_{2}+\nabla u_{3} \cdot \nabla v_{3}\right\}+\mathbf{u}^{T} \mathbf{v} d \mathbf{x} .
$$


and norm

$$
\|\mathbf{v}\|_{V}=(\mathbf{v}, \mathbf{v})_{V}^{\frac{1}{2}}
$$

To include a weak version of the divergence free condition (2.8), define the bilinear form

$$
(\mathbf{u}, \mathbf{v})_{1}=\int_{\Omega}[\nabla \cdot \mathbf{u}][\nabla \cdot \mathbf{v}] d \mathbf{x} .
$$

on the Hilbert space $\mathbf{V}$.

Remark 2.4. Note that a norm equivalent to (2.10), see [5], on $\mathbf{V}$ may be obtained using the bilinear form

$$
((\mathbf{u}, \mathbf{v}))=\int_{\Omega}\left\{\nabla u_{1} \cdot \nabla v_{1}+\nabla u_{2} \cdot \nabla v_{2}+\nabla u_{3} \cdot \nabla v_{3}\right\} d \mathbf{x} .
$$

to which is added

$$
\int_{\Omega} \mathbf{u}^{T}\left[\phi_{0}^{2} \mathbf{r}_{0} \mathbf{r}_{0}^{T}\right] \mathbf{v} d \mathbf{x}
$$

where the subscript " 0 " is an index for the point $\mathbf{x}_{0}$ that is the site of a radar from which measurements are made. The quantity

$$
\left\{((\mathbf{v}, \mathbf{v}))+K_{0} \int_{\Omega} \mathbf{v}(\mathbf{x})^{T}\left[\phi_{0}^{2} \mathbf{r}_{0} \mathbf{r}_{0}^{T}\right] \mathbf{v} d \mathbf{x}\right\}^{\frac{1}{2}}
$$

where $K_{0}$ is a positive number is a norm on $\mathbf{V}$ equivalent to that of $(2.10)$ (ii).

The weak formulation of the retrieval problem is posed as a minimization problem over the space $\mathbf{V}$ with the objective functional given by

$$
\begin{gathered}
\mathcal{V}(\mathbf{v})=\frac{\epsilon}{2}((\mathbf{v}, \mathbf{v}))+\frac{K}{2}(\mathbf{v}, \mathbf{v})_{1}+ \\
+\frac{K_{1}}{2} \int_{\Omega}\left\{\sum_{i=1}^{n} \phi_{i}^{2}(\mathbf{x})\left[v_{r}\left(\mathbf{x}, \mathbf{x}_{i}\right)-v_{r i}(\mathbf{x})\right]^{2}\right\} d \mathbf{x}
\end{gathered}
$$

where $\epsilon, K$, and $K_{1}$ are positive constants. The retrieval problem is thus

$$
\text { Find } \mathbf{u} \in \mathbf{V} \text { such that } \mathcal{V}(\mathbf{u})=\operatorname{infimum}\{\mathcal{V}(\mathbf{v}): \mathbf{v} \in \mathbf{V}\}
$$

Remark 2.5. Note that time is not explicitly included. In this formulation the retrieval problem is solved over a sequence of times. It is assumed that the radial velocity is known at each point $\mathbf{x}$ within a given radar's scanning set at each time. 
Our interest here will focus on the dependence of solutions of the retrieval problem on the collection of radar scanning directions. Hence, we view the vector

$$
q=\left[\begin{array}{llll}
\alpha_{1} & \alpha_{2} & \ldots & \alpha_{n}
\end{array}\right]
$$

as a parameter to be determined. Define the functions

$$
\Phi(q)(\mathbf{x})=\sum_{i=1}^{n} \phi_{i}\left(\mathbf{x}, \alpha_{i}\right) \mathbf{r}_{i}(\mathbf{x}) \mathbf{r}_{i}^{T}(\mathbf{x}),
$$

the vector-valued functions

$$
\mathbf{F}(q)(\mathbf{x})=\sum_{i=1}^{n} \phi_{i}\left(\mathbf{x}, \alpha_{i}\right)\left(\mathbf{r}_{i}^{T} \mathbf{v}_{t}(\mathbf{x})+v_{r_{i}}(\mathbf{x}) \mathbf{r}_{i}\right)
$$

and the constants

$$
C(q)=\int_{\Omega}\left\{\sum_{i=1}^{n} \phi_{i}\left(\mathbf{x}, \alpha_{i}\right)\left(\mathbf{r}_{i}(\mathbf{x})^{T} \mathbf{v}_{t}(\mathbf{x})-v_{r_{i}}(\mathbf{x})\right)^{2}\right\} d \mathbf{x} .
$$

Note that $\mathbf{F}(q)$ depends on $q$, not only through $\phi_{i}$ and $\mathbf{r}_{i}$, but also $v_{r i}$.

It is also convenient to define the family of bilinear forms on $\mathbf{H}$ parameterized by $q$ given by

$$
(\mathbf{u}, \mathbf{v})_{\Phi(q)}=\int_{\Omega} \mathbf{u}(\mathbf{x})^{T} \Phi(q)(\mathbf{x}) \mathbf{v}(\mathbf{x}) d \mathbf{x}
$$

With the above definitions, we may write the criterion $\mathcal{V}$ as

$$
\begin{gathered}
\mathcal{V}(q)(\mathbf{v})=\frac{\epsilon}{2}((\mathbf{v}, \mathbf{v}))+\frac{K}{2}(\mathbf{v}, \mathbf{v})_{1}+\frac{K_{1}}{2}(\mathbf{v}, \mathbf{v})_{\Phi(q)}- \\
\left.-K_{1}(\mathbf{F}(q), \mathbf{v})\right] \frac{K_{1}}{2} C(q) .
\end{gathered}
$$

Existence of a unique solution to the minimization problem (2.15) follows from the discussions in [14].

Proposition 2.6. There exists a unique solution to the minimization problem (2.15).

For each parameter vector $q$ there exists a unique solution. Thus, we set $\mathbf{u}(q)=$ $\mathbf{u}$ where $\mathbf{u}$ is the solution of (2.15) for the parameter $q$. The solution of the minimization problem (2.15) is characterized by the optimality conditions. 
Proposition 2.7. The Fréchet derivative of $\mathcal{V}$ with respect to $\mathbf{u}$ is given by

$$
D \mathcal{V}(q)(\mathbf{u}) \mathbf{v}=\epsilon((\mathbf{u}, \mathbf{v}))+K(\mathbf{u}, \mathbf{v})_{1}+K_{1}(\mathbf{u}, \mathbf{v})_{\Phi(q)}-K_{1}(\mathbf{F}(q), \mathbf{v})
$$

and the solution $\mathbf{u}=\mathbf{u}(q)$ of the minimization problem (2.15) satisfies the equation

$$
\epsilon((\mathbf{u}, \mathbf{v}))+K(\mathbf{u}, \mathbf{v})_{1}+K_{1}(\mathbf{u}, \mathbf{v})_{\Phi(q)}=K_{1}(\mathbf{F}(q), \mathbf{v})
$$

for all $\mathbf{v} \in \mathbf{V}$. When the data is associated with a "true" wind field $\mathbf{w}$ so that $(\mathbf{F}(q), \mathbf{v})=(\mathbf{w}, \mathbf{v})_{\Phi(q)}$, we have

$$
\epsilon((\mathbf{u}, \mathbf{v}))+K(\mathbf{u}, \mathbf{v})_{1}+K_{1}(\mathbf{u}, \mathbf{v})_{\Phi(q)}=K_{1}(\mathbf{w}, \mathbf{v})_{\Phi(q)}
$$

Remark 2.8. The retrieved wind field satisfies the estimate

$$
\|\mathbf{u}(q)\|_{V} \leq \text { Constant }\|\mathbf{F}(q)\|_{H}
$$

where the "Constant" depends on $\epsilon, K$, and $K_{1}$.

To obtain the retrieved wind field in an application requires an approximation of the solution of (2.21). Towards this end, we approximate (2.21) by finite elements. The approximation of the retrieval problem numerically follows the classical finite element arguments [8]. Approximations may be based on finite elements obtained as tensor products of piecewise linear splines defined on partitions of the intervals $\left(0, L_{x}\right),\left(0, L_{y}\right)$, and $\left(0, L_{z}\right)$ into $n_{x}, n_{y}$, and $n_{z}$ subintervals, respectively. Hence, setting $m_{x}=n_{x}+1, m_{y}=n_{y}+1, m_{z}=n_{z}+1$ to represent the number of $x, y$, and $z$ elements, respectively, the number of basis elements for the 3 spatial dimensional problem is given by $m=m_{x} \times m_{y} \times m_{z}$. We denote the basis elements as

$$
b_{1}(\mathbf{x}), \ldots, b_{m}(\mathbf{x})
$$

spanning a subspace, $V^{m}$, of the space $V$. Define the column $m$ vector-valued function on $\Omega$ by

$$
\mathbf{x} \mapsto \underline{b}(\mathbf{x})=\left[b_{1}(\mathbf{x}), \ldots, b_{m}(\mathbf{x})\right]^{T}
$$

and the $3 \times 3 m$ matrix-valued function on $\Omega$ by

$$
\mathbf{x} \mapsto B(\mathbf{x})=\left[\begin{array}{ccc}
\underline{b}(\mathbf{x})^{T} & \underline{0} & \underline{0} \\
\underline{0} & \underline{b}(\mathbf{x})^{T} & \underline{0} \\
\underline{0} & \underline{0} & \underline{b}(\underline{\mathbf{x}})^{T}
\end{array}\right]
$$

where $\underline{0}$ represents an $\mathrm{m}$-row vector of zeros. We also define the column $\mathrm{m}$ vectors $\underline{c}_{1}, \underline{c}_{2}$, and $\underline{c}_{3}$ as well as the $3 \mathrm{~m}$-column vector $\widetilde{c}=\left[\underline{c}_{1}^{T}, \underline{c}_{2}^{T}, \underline{c}_{3}^{T}\right]^{T}$.

With the above definitions, we represent the components of the wind velocity as

$$
v_{1 m}(\mathbf{x})=\underline{b}(\mathbf{x})^{T} \underline{c}_{1}
$$




$$
v_{2 m}(\mathbf{x})=\underline{b}(\mathbf{x})^{T} \underline{c}_{2}
$$

and

$$
v_{3 m}(\mathbf{x})=\underline{b}(\mathbf{x})^{T} \underline{c}_{3} .
$$

Let $\mathbf{V}^{m}=V^{m} \times V^{m} \times V^{m}$ designate the analogous subspace of $\mathbf{V}$. The approximating wind velocity vector $\mathbf{v}_{\mathbf{m}}$ is expressed as

$$
\mathbf{v}_{m}(\mathbf{x})=\left[v_{1 m}(\mathbf{x}) v_{2 m}(\mathbf{x}) v_{3 m}(\mathbf{x})\right]^{T}=B(\mathbf{x}) \widetilde{c} .
$$

To facilitate the expression of the approximating equations, define the $3 m \times$ $3 m$ matrices

$$
\begin{gathered}
G_{0}=\int_{\Omega} B(\mathbf{x})^{T} B(\mathbf{x}) d \mathbf{x} \\
G_{1}=\int_{\Omega}\left[\nabla^{T} B(\mathbf{x})\right]^{T}\left[\nabla^{T} B(\mathbf{x})\right] d \mathbf{x} .
\end{gathered}
$$

Further, define the $m \times m$ matrix $g_{2}$ by setting entries

$$
\left(g_{2}\right)_{i j}=\int_{\Omega} \nabla b_{i}(\mathbf{x}) \cdot \nabla b_{j}(\mathbf{x}) d \mathbf{x}
$$

for $i, j=1, \ldots, m$. Let the $3 m \times 3 m$ matrices be given by

$$
\begin{gathered}
G_{2}=\left[\begin{array}{ccc}
g_{2} & 0 & 0 \\
0 & g_{2} & 0 \\
0 & 0 & g_{2}
\end{array}\right] . \\
G(q)=\int_{\Omega} B(\mathbf{x})^{T} \Phi(q) B(\mathbf{x}) d \mathbf{x} .
\end{gathered}
$$

Finally, define the $3 m$ column vector

$$
\widetilde{F}(q)=\left\{\int_{\Omega} \mathbf{F}(q)(\mathbf{x})^{T} B(\mathbf{x}) d \mathbf{x}\right\}^{T} .
$$

With these definitions the objective functional evaluated at the finite element approximations of the wind velocity is given by

$(2.24) \mathcal{V}(q)(\widetilde{c})=\mathcal{V}(q)\left(\mathbf{v}^{m}\right)=\frac{1}{2} \widetilde{c}^{T}\left[\epsilon G_{2}+K G_{1}+K_{1} G(q)\right] \widetilde{c}-K_{1} \widetilde{F}(q)^{T} \widetilde{c}+C(q)$.

Remark 2.9. The finite dimensional minimization problem looks for a function of the form

$$
\mathbf{u}^{m}=\mathbf{u}^{m}(q)=B(\mathbf{x}) \widetilde{c}=B(\mathbf{x}) \widetilde{c}(q)
$$

in $\mathbf{V}^{m}$ minimizing the functional $\mathcal{V}(q)$ over $\mathbf{V}^{m}$.

The derivative of this functional gives the system

$$
\epsilon\left(\left(\mathbf{u}^{m}, \mathbf{v}\right)\right)+K\left(\mathbf{u}^{m}, \mathbf{v}\right)_{1}+K_{1}\left(\mathbf{u}^{m}, \mathbf{v}\right)_{\Phi(q)}=
$$




$$
=K_{1}(\mathbf{F}(q), \mathbf{v})
$$

for all $\mathbf{v} \in \mathbf{V}^{m}$. In terms of matrices the solution vector $\widetilde{c}$ satisfies

$$
\left[\epsilon G_{2}+K G_{1}+K_{1} G(q)\right] \widetilde{c}=K_{1} \widetilde{F}(q)
$$

Error between $\mathbf{u}$ and $\mathbf{u}^{m}$ is estimated by taking the difference of (2.21) and (2.25) so that

$$
\epsilon\left(\left(\mathbf{u}-\mathbf{u}^{m}, \mathbf{v}\right)\right)+K\left(\mathbf{u}-\mathbf{u}^{m}, \mathbf{v}\right)_{1}+K_{1}\left(\mathbf{u}-\mathbf{u}^{m}, \mathbf{v}\right)_{\Phi(q)}=0
$$

for any $\mathbf{v} \in \mathbf{V}^{m}$. Thus, we find that

$$
\begin{gathered}
\epsilon\left(\left(\mathbf{u}-\mathbf{u}^{m}, \mathbf{u}-\mathbf{u}^{m}\right)\right)+K\left(\mathbf{u}-\mathbf{u}^{m}, \mathbf{u}-\mathbf{u}^{m}\right)_{1}+K_{1}\left(\mathbf{u}-\mathbf{u}^{m}, \mathbf{u}-\mathbf{u}^{m}\right)_{\Phi(q)}= \\
\quad=\epsilon\left(\left(\mathbf{u}-\mathbf{u}^{m}, \mathbf{u}-\mathbf{v}\right)\right)+K\left(\mathbf{u}-\mathbf{u}^{m}, \mathbf{u}-\mathbf{v}\right)_{1}+K_{1}\left(\mathbf{u}-\mathbf{u}^{m}, \mathbf{u}-\mathbf{v}\right)_{\Phi(q)}
\end{gathered}
$$

and

$$
\left\|\mathbf{u}-\mathbf{u}^{m}\right\|_{V}^{2} \leq \text { Constant }\left\|\mathbf{u}-\mathbf{u}^{m}\right\|_{V}\|\mathbf{u}-\mathbf{v}\|_{V}
$$

so that

$$
\left\|\mathbf{u}-\mathbf{u}^{m}\right\|_{V} \leq \text { Constant }\|\mathbf{u}-\mathbf{v}\|_{V}
$$

for any $\mathbf{v} \in \mathbf{V}^{m}$.

Remark 2.10 The rate of convergence thus depends on the regularity of the solution $\mathbf{u}$.

Finally, we approximate $\mathbf{w}=B(\mathbf{x}) \widetilde{\omega}$ and note the finite dimensional approximating system of equations of $(2.22)$ is given by

$$
\left(\epsilon G_{2}+K G_{1}+K_{1} G(q)\right) \widetilde{c}(q)=K_{1} G(q) \widetilde{\omega}
$$

\section{Optimal Retrieval based on an Ensemble Rel- ative Error Criterion.}

In Section 2 the retrieval problem is formulated as a minimization problem estimating a three dimensional wind field from radar data conditioned with physics-based side constraints. The solution of the retrieval problem (2.15) depends on the scanning region for each radar site. For a network consisting of $\mathrm{n}$ radars located at points $x_{1}, x_{2}, \ldots, x_{n}$, the collection of scanning directions may be expressed as an $\mathrm{n}$ vector $q=\left(\alpha_{1}, \alpha_{2}, \ldots, \alpha_{n}\right)$ of real numbers $\alpha_{i} \in[-\pi, \pi)$. The objective here is to study the properties of the retrieved wind fields with 
respect to radar scanning directions. These properties are then extended to retrieval operators that describe the mapping taking a given wind field to its corresponding retrieved wind field. Given an ensemble of meteorological events, a relative error function is defined that compares retrieved wind fields with the associated test fields in the ensemble. The relative retrieval error is a function of the scanning parameters used in specifying the observation operator. It is possible then to determine those scanning parameters that minimize the relative error functional.

We begin by establishing differentiability with respect to the direction angle parameters. Towards this end, let the function $\mathbf{x} \mapsto g(\mathbf{x})$ be a continuous realvalued function defined on $\Omega$. For a single parameter $\alpha$, define the real-valued function $\mathcal{F}: \Re \mapsto \Re$ in terms of the integral given by

$$
\mathcal{F}(\alpha)=\int_{\Omega} \Xi(\mathbf{x}, \alpha) g(\mathbf{x}) d \mathbf{x}
$$

where $\Xi(\cdot, \alpha)$ is the indicator function of the set

$$
C(\alpha)=\left\{\mathbf{x}: x_{1}^{2}+x_{2}^{2} \leq R^{2}, \frac{\mathbf{x}}{|\mathbf{x}|} \cdot \mu(\alpha) \geq \cos (\beta)\right\}
$$

and $\mathbf{x}=\left[x_{1} x_{2} x_{3}\right]^{T}$ Thus,

$$
\mathcal{F}(\alpha)=\int_{C(\alpha)} g(\mathbf{x}) d \mathbf{x}
$$

Introducing polar coordinates, we see that

$$
\mathcal{F}(\alpha)=\int_{\alpha-\beta}^{\alpha+\beta} \int_{0}^{L_{z}} \int_{0}^{R} \widehat{g}(r, \theta, z) r d r d z d \theta
$$

where $\widehat{g}(r, \theta, z)=g(r \cos (\theta), r \sin (\theta), z)$. It follows immediately that

$$
\frac{d}{d \alpha} \mathcal{F}(\alpha)=\int_{0}^{L_{z}} \int_{0}^{R}[\widehat{g}(r, \alpha+\beta, z)-\widehat{g}(r, \alpha-\beta, z)] r d r d z .
$$

For the case of $\mathrm{n}$ radar sites, we write

$$
\begin{gathered}
\mathcal{F}(\mathbf{u}, \mathbf{v})\left(\alpha_{1}, \ldots, \alpha_{n}\right)=(\mathbf{u}, \mathbf{v})_{\Phi(q)} \\
=\int_{\Omega} \mathbf{u}(\mathbf{x})^{T}\left[\sum_{i=1}^{n} \phi_{i}\left(\mathbf{x}, \alpha_{i}\right) \mathbf{r}_{i}(\mathbf{x}) \mathbf{r}_{i}^{T}(\mathbf{x}] \mathbf{v}(\mathbf{x}) d \mathbf{x} .\right.
\end{gathered}
$$

Setting

$$
g_{l}(\mathbf{x})=\phi_{l}\left(\mathbf{x}, \alpha_{l}\right) \mathbf{u}(\mathbf{x})^{T}\left[\mathbf{r}_{l}(\mathbf{x}) \mathbf{r}_{l}^{T}(\mathbf{x})\right] \mathbf{v}(\mathbf{x})
$$

we see that

$$
\mathcal{F}(\mathbf{u}, \mathbf{v})\left(\alpha_{1}, \ldots, \alpha_{n}\right)=\sum_{i=1}^{n} \int_{\Omega} g_{i}(\mathbf{x}) d \mathbf{x}
$$


and we have the following.

Proposition 3.1. For each $\mathbf{u}$ and $\mathbf{v} \in \mathbf{V}$ the function $\left(\alpha_{1}, \ldots, \alpha_{n}\right) \mapsto \mathcal{F}(\mathbf{u}, \mathbf{v})\left(\alpha_{1}, \ldots, \alpha_{n}\right)$ is differentiable and the partial derivatives of $\mathcal{F}(\mathbf{u}, \mathbf{v})$ with respect to $\alpha_{l}$ is given by

$$
\frac{\partial}{\partial \alpha_{l}} \mathcal{F}\left(\alpha_{1}, \ldots, \alpha_{n}\right)=\int_{0}^{L_{z}} \int_{0}^{R}\left[g_{l}\left(r, \alpha_{l}+\beta, z\right)-g_{l}\left(r, \alpha_{l}-\beta, z\right)\right] r d r d z
$$

The differentiability of $\mathbf{u}(q)$ follows.

Theorem 3.2. The solution $\mathbf{u}(q)$ of $(2.22)$ is Fréchet differentiable with respect to $q$ and satisfies

$$
\begin{gathered}
\epsilon\left(\left(D \mathbf{u}(q) q^{\prime}, \mathbf{v}\right)\right)+K\left(D \mathbf{u}(q) q^{\prime}, \mathbf{v}\right)_{1}+K_{1}\left(D \mathbf{u}(q) q^{\prime}, \mathbf{v}\right)_{\Phi(q)}= \\
=K_{1}\left[D \mathcal{F}(\mathbf{w}, \mathbf{v})(q) q^{\prime}-D \mathcal{F}(\mathbf{u}(q), \mathbf{v})(q) q^{\prime}\right]
\end{gathered}
$$

where $\mathcal{F}(\mathbf{w}, \mathbf{v})(q)=(\mathbf{w}, \mathbf{v})_{\Phi(q)}$

We also note that the finite dimensional version satisfies

Corollary 3.3. The derivative $D \widetilde{c}(q)\left(q^{\prime}\right)$ of the solution $\widetilde{c}(q)$, of equation (2.26) satisfies the equation

$$
\left[\epsilon G_{2}+K G_{1}+K_{1} G(q)\right] D \widetilde{c}(q) q^{\prime}=K_{1}\left[D G(q) q^{\prime}\right][\widetilde{\omega}-\widetilde{c}(q)]
$$

The Fréchet differentiability result of Theorem 3.2, of course, implies the continuous dependence of retrieved wind fields on the scanning angle vector $q$ subject to continuous dependence of the observations $v_{r}$ [4]. The result depends on the character of the observations. In [14] to measure the effectiveness of a retrieval algorithm, procedures are tested against a variety of possible observed data. Data are constructed using an observation operator and a hypothesized wind field. Given a hypothetical three dimensional wind field $\mathbf{w}_{0} \in \mathbf{V}$, we define the vector-valued function $\mathbf{F}(q)$ to be

$$
\mathbf{F}(q)(\mathbf{x})=\Phi(q)(\mathbf{x})\left[\mathbf{w}_{0}(\mathbf{x})+\mathbf{v}_{t}(\mathbf{x})\right]
$$

where $\Phi(q)(\mathbf{x})$ is given by equation (2.16). Setting $\mathbf{w}=\mathbf{w}_{0}+\mathbf{v}_{t}$ it follows that for any $\mathbf{v} \in \mathbf{V}$ and

$$
(\mathbf{F}(q), \mathbf{v})=(\mathbf{w}, \mathbf{v})_{\Phi(q)}
$$

obtaining equation (2.22). Continuity properties of the mapping $q \mapsto \mathbf{u}(q)$ are given in the following results.

Proposition 3.4. Let $\mathbf{w}$ be an element of $\mathbf{V}$ and let the sequence of vectors $\left\{q_{k}\right\}_{k=1}^{\infty}$ converge componentwise to the vector $q$ as $k \rightarrow \infty$, then the sequence 
$\left\{\mathbf{u}\left(q_{k}\right)\right\}_{k=1}^{\infty}$ of solutions to the associated retrieval problems converges to $\mathbf{u}(q)$ in $\mathbf{V}$.

The solution $\mathbf{u}(q)=\mathbf{u}$ of (2.22) defines a bounded linear operator that we refer to as the retrieval operator and designate as $R(q): \mathbf{V} \mapsto \mathbf{V}$ by

$$
R(q) \mathbf{w}=\mathbf{u}(q) .
$$

Convergence stated in terms of the retrieval operator is given by the following.

Proposition 3.5. Suppose $q^{k} \longrightarrow q$ as $k \longrightarrow \infty$ in the sense that the entries of $q^{k}$ converge to the entries of $q$. Then for each $\mathbf{w} \in \mathbf{V}$

$$
R\left(q^{k}\right) \mathbf{w} \longrightarrow R(q) \mathbf{w}
$$

in $\mathbf{V}$. For the finite dimensional case (2.28), we have

$$
\widetilde{c}(q)=K_{1}\left[\epsilon G_{2}+K G_{1}+K_{1} G(q)\right]^{-1} G(q) \widetilde{\omega}
$$

For the finite dimensional formulation, define the retrieval operator

$$
R^{m}(q) \widetilde{\omega}=\widetilde{c}(q)
$$

and the finite dimensional approximation to the retrieved solution is then given by

$$
\mathbf{u}^{m}(q)(\mathbf{x})=B(\mathbf{x}) \widetilde{c}(q)=B(\mathbf{x}) R^{m}(q) \widetilde{\omega} .
$$

To optimize the retrieval procedure, an objective functional is defined with which to compare different scanning vectors. In this work we consider the relative retrieval error. To define the relative retrieval error, suppose a parameter $q \in Q$. Also, assume a wind field $\mathbf{w} \in \mathbf{V}$. The retrieved wind field discussed above is expressed as $\mathbf{u}=\mathbf{u}(q)=R(q) \mathbf{w}$. The relative error is given by

$$
J(q)=\frac{\|R(q) \mathbf{w}-\mathbf{w}\|^{2}}{\|\mathbf{w}\|^{2}}
$$

with the finite dimensional relative error expressed as

$$
J^{m}(q)=\frac{\left(R^{m}(q) \widetilde{\omega}-\widetilde{\omega}\right)^{T} G_{0}\left(R^{m}(q) \widetilde{\omega}-\widetilde{\omega}\right)^{T}}{\widetilde{\omega} G_{0} \widetilde{\omega}^{T}}
$$

Let $Q_{a d}$ be an admissible subset of parameters in $Q$. For the given a wind field $\mathbf{w}$ in $\mathbf{V}$, optimizing the retrieval procedure for $\mathbf{w}$ seeks to determine a parameter $q_{o}$ from from within the set $Q_{a d}$ to minimize the relative retrieval error $J(q)$. The problem is more succinctly stated as follows. 
Suppose $\mathbf{w} \in \mathbf{V}$ and $Q_{a d}$ is a compact subset of $Q$.

Find $q_{o} \in Q_{a d}$ such that

$$
J\left(q_{o}\right)=\operatorname{infimum}\left\{J(q): q \in Q_{a d}\right\} .
$$

From the continuous dependence on $q$, we have the following.

Theorem 3.8. For each $\mathbf{w} \in V$ there exists a a solution to problem (3.8).

Proof. From Proposition 3.5, given $\mathbf{w} \in H$ the mapping $q \mapsto R(q) \mathbf{w}$ is continuous from $Q_{a d}$ into $\mathbf{V}$. Thus, the function $q \mapsto J(q)$ is a continuous real valued nonnegative function from $Q_{a d}$ into $\Re$. Since $Q_{a d}$ is compact, the existence of a minimizer follows immediately.

The solution $q_{o}$ clearly depends on the test wind field $\mathbf{w}$. Thus, the radar scanning vector can be chosen to be optimal with respect to a prescribed subclass of wind fields. Suppose then that $\mathbf{W}=\left\{\mathbf{w}_{n}: n=1, \ldots, N\right\}$ is a collection of wind fields. Let $p_{n}, n=1, \ldots, N$ be weights such $p_{n}>0$, and $\sum_{n=1}^{N} p_{n}=1$. We consider then the optimization problem based on the functional

$$
J(q)=\sum_{n=1}^{N} p_{n} \frac{\left\|R(q) \mathbf{w}_{n}-\mathbf{w}_{n}\right\|_{H}^{2}}{\left\|\mathbf{w}_{n}\right\|_{\mathbf{H}}^{2}} .
$$

Existence of a solution then follows from Proposition 3.7.

The proof of convergence of solutions of the finite dimensional approximating problems is analogous to that in [14]. Let

$$
J^{m}(q)=\sum_{i=1}^{N} p_{i} \frac{\left\|\mathbf{u}^{m}(q)-\mathbf{w}_{i}\right\|_{H}^{2}}{\left\|\mathbf{w}_{i}\right\|_{V}^{2}}
$$

and let $q^{m}$ be such that

$$
J^{m}\left(q^{m}\right)=\inf \left\{J^{m}(q): q \in Q_{a d}\right\}
$$

Note that the sequence $q^{m}$ has cluster points since $Q_{a d}$ is compact.

Theorem 3.9. Every cluster point $q_{o}$ of the sequence of parameters $q^{m}$ is a solution of (3.8).

Proof. Let $q^{m} \longrightarrow q_{o}$, then $q_{o} \in Q_{a d}$. Then $\mathbf{u}^{m}\left(q^{m}\right)$ is bounded in $\mathbf{V}$ from the a priori estimates. From weak compactness, it follows that there is a subsequence, again $\mathbf{u}^{m}\left(q^{m}\right)$, that converges weakly in $\mathbf{V}$ to $\mathbf{u}$. That $\mathbf{u}=\mathbf{u}\left(q_{o}\right)$ follows from the variational condition (2.25), the compact embedding of $\mathbf{V}$ in $L^{4}\left(\Omega, \Re^{3}\right),[2]$, and uniqueness. Further, convergence of the sequence $\mathbf{u}^{m}\left(q^{m}\right) \longrightarrow \mathbf{u}\left(q_{o}\right)$ weakly in $\mathbf{V}$ and strongly in $\mathbf{H}$ follows. 
Let $\widetilde{q}$ be an arbitrary element in $Q_{a d}$. From the optimality of $q^{m}$, it follows that

$$
J^{m}\left(q^{m}\right) \leq J^{m}(\widetilde{q}) .
$$

From the above convergence properties it follows then that

$$
J\left(q_{o}\right) \leq J(\widetilde{q}) .
$$

Since $\widetilde{q}$ is an arbitrary element of $Q_{a d}$ and $q_{o}$ belongs to $Q_{a d}$, it follows that $q_{o}$ is a minimizer.

The selection of the class $\mathbf{W}$ can be used to emphasize certain significant wind fields that are deemed to be most likely, see [14]. Alternatively, $\mathbf{W}$ might be chosen randomly. In any case it is useful to think of $\mathbf{W}$ as a test set of significant wind fields of interest. In the radar scanning problem, consider $\mathbf{W}$ to be chosen to capture uncertainty in wind fields propagated in a time stepping model from previous estimated wind fields. Hence, we assume a prediction model of the form

$$
\mathbf{w}=\mathbf{F}_{M}\left(\mathbf{w}_{o}\right)
$$

where $\mathbf{F}_{M}: \Re^{3} \mapsto \Re^{3}$ is continuously differentiable. Typically, $\mathbf{w}_{o}$ represents a current estimate of wind fields. The model then predicts the wind field at a subsequent time to be $\mathbf{w}$, and thus, is a function of $\mathbf{w}_{o}$. For implementation, we replace equation (3.10) with a discrete version

$$
\widetilde{\omega}=\mathcal{F}_{M}\left(\widetilde{\omega}_{o}\right)
$$

where

$$
\mathbf{w}=B(\mathbf{x}) \widetilde{\omega}
$$

and

$$
\mathbf{w}_{o}=B(\mathbf{x}) \widetilde{\omega}_{o} .
$$

The Fré chet derivative is then given by

$$
D \widetilde{\omega}\left(\widetilde{\omega}_{o}\right) \widetilde{\omega}^{\prime}=D \mathcal{F}_{M}\left(\widetilde{\omega}_{o}\right) \widetilde{w}^{\prime} .
$$

Set

$$
S(q)=[R(q)-I]^{T} G_{0}[R(q)-I]
$$

and consider perturbations $\widetilde{\delta}_{k}$ for $k=1, \ldots, N$ of $\widetilde{\omega}_{o}$. Possible functionals are given by

$$
J(q)=\sum_{k=1}^{N} p_{k} \frac{\mathcal{F}_{M}\left(\widetilde{\omega}_{o}+\widetilde{\delta}_{k}\right)^{T} S(q) \mathcal{F}_{M}\left(\widetilde{\omega}_{o}+\widetilde{\delta}_{k}\right)}{\mathcal{F}_{M}\left(\widetilde{\omega}_{o}+\widetilde{\delta}_{k}\right)^{T} G_{0} \mathcal{F}_{M}\left(\widetilde{\omega}_{o}+\widetilde{\delta}_{k}\right)}
$$

and

$$
J_{0}(q)=\sum_{k=1}^{N} p_{k} \frac{\left[\mathcal{F}_{M}\left(\widetilde{\omega}_{o}\right)+D \mathcal{F}_{M}\left(\widetilde{\omega}_{o}\right) \delta_{k}\right]^{T} S(q)\left[\mathcal{F}_{M}\left(\widetilde{\omega}_{o}\right)+D \mathcal{F}_{M}\left(\widetilde{\omega}_{o}\right) \delta_{k}\right]}{\left.\left[\widetilde{\mathcal{\omega}}_{o}\right)+D \mathcal{F}_{M}\left(\widetilde{\omega}_{o}\right) \delta_{k}\right]^{T} G_{0}\left[\mathcal{F}_{M}\left(\widetilde{\omega}_{o}\right)+D \mathcal{F}_{M}\left(\widetilde{\omega}_{o}\right) \delta_{k}\right]}
$$

In the next section we consider the functional (3.13) for a simple wind field model describing a vortex moving across the observation region with two radars. 


\section{Numerical Study.}

We propose the following general algorithmic procedure for scanning optimization.

1. Input estimated wind field.

2. Generate an ensemble of perturbed wind fields about the estimated.

3. Advance the model for each perturbed wind field to obtain an ensemble of predicted fields.

4. Generate an admissible scan parameter.

5. Calculate a retrieved wind field based on that scan parameter for each of the ensemble of perturbed wind fields.

6. Calculate a relative error between the retrieved and the model wind fields over the ensemble of perturbations.

7. Select scan parameter minimizing the relative error functional.

8. Use the selected scan parameter to make actual radar measurements.

9. Based on the radar measurements estimate wind fields.

10. Return to 1 . and repeat the procedure.

To start the procedure the initial estimated wind field may be taken to be the zero wind field. Thus, the ensemble of random fields obtained as perturbations of the zero wind field is used to start the procedure. With an ensemble of randomly generated fields, any direction is as good as any other direction for scanning. Thus, in the initial stages sitting and spinning of the radar can be considered to be optimal. In this instance one may observe that the relative error is a constant function of the scanning vector. When the functional is no longer constant an optimal scanning direction may be determined. It is then used to as the direction in which the radars are pointed to actually collect data. The retrieved wind field based on that scanning vector is the estimated wind field to be used in the beginning of the next iteration. Perturbations of that estimate are advanced using the model to construct the ensemble of likely wind fields. The procedure repeats determining the scanning direction that is optimal with respect to that ensemble. Although it is stated that the optimal scanning parameters are used, in the example present here the optimization is with respect to small admissible set of sixteen directions, four possible directions for each radar site. Optimization with respect to larger and more complicated admissible sets of scanning vectors will be considered in subsequent work. 
To construct an example, we begin by specifying a wind field evolving with time that models a vortex event moving across the observational region. The resulting wind field serves as "truth" to be observed with various scanning strategies. It is necessary to specify the path as a function of time along with a vector field modelling the vortex. This is superimposed on a background that for this example is zero. The center of the generated test vortex is given by

$$
\mathbf{x}_{0}(t)=[10 t, 10 t+10,0]^{T}
$$

The wind field at time $t$ is obtained by

$$
\mathbf{u}(\mathbf{x}, t)=\left[-\frac{\partial \psi}{\partial x_{2}}, \frac{\partial \psi}{\partial x_{1}}, 0\right]
$$

where

$$
\psi(\mathbf{x}, t)=-\exp \left[\left(\frac{\left|\mathbf{x}-\mathbf{x}_{0}(t)\right|}{R}-1\right)^{-1}\right]
$$

In the example the time parameter takes integer values $t=0,1, \ldots, 15$. For the purpose of the numerical experiment we use the model

$$
\mathbf{u}(\mathbf{x}, t+d t)=\mathbf{u}(\mathbf{x}, t)+d t \frac{\partial \mathbf{u}(\mathbf{x}, t)}{\partial t}
$$

In terms of the model function define

$$
\mathbf{F}_{M}(\mathbf{u}(\mathbf{x}, t))=\mathbf{u}(\mathbf{x}, t)+d t \frac{\partial \mathbf{u}(\mathbf{x}, t)}{\partial t}
$$

As described in the above procedure, we start with an initial estimate of wind velocity being zero over the observational domain. To capture uncertainty in the estimate and the direction of propagation the estimate is randomly perturbed along with the partial derivative to obtain an ensemble of possible wind fields at the next step. For a given scanning vector, a retrieved estimate is obtained for each member of the ensemble. The resulting collection of retrieved wind field is compared with the ensemble members by means of the relative retrieval error functional. Hence, the relative retrieval error is a function of the given scanning vector. An optimal scanning vector is determined by selecting that scanning vector minimizing the relative retrieval functional. In the present example, the admissible set consists of only 16 direction vectors, 4 for each radar site. It is possible to conduct minimization by exhaustively searching the admissible set at each step.

In Figures 1 through 15 are portrayed the scanning location and coverage of each radar is indicated by the shaded region. Also, the vortex location and wind directions are easily noted. The vectors that are out of line with the vortex vectors are estimated wind vectors. It can be seen that wind vectors 
are estimated even outside of the coverage regions if some portion of the vortex intersects the coverage region. This is due to the weak inclusion of the divergence condition. If there is no intersection with the coverage region, the estimate is zero, and the radar coverage is random. In this example, the coverage anticipates the next location of the vortex and adjusts the coverage appropriately. The exception is in Figure 12. In Figure 13 adjustment is made that seems more reasonable.

Acknowledgement This work is supported in part by the Engineering Research Centers Program of the National Science Foundation under NSF Award Number (EEC-0313747). Any opinions, findings and conclusions or recommendations expressed in this material are those of the authors and do not necessarily reflect those of the National Science Foundation. 


\section{References}

[1] C. H. Bishop, B. J. Etherton, and S. J. Majumdar, "Adaptive Sampling with the Ensemble Transform Kalman Filter. Part I: Theoretical Aspects," Monthly Weather Review Vol. 129, pp.420-436, 2001.

[2] D. Gilbarg and N.S. Trudinger, Elliptic Partial Differential Equations of Second Order, 2nd Edition, Springer-Verlag, New York, 1983.

[3] J. L. Lions, Distributed Optimal Control of Systems Governed by Partial Differential Equations, Springer-Verlag, New York, 1969.

[4] D.G. Luenberger, Optimization by Vector Space Methods, Wiley, New York, 1969.

[5] V.G. Max'ja,Sobolev Spaces, Springer-Verlag, New York, 1985.

[6] John Mewes and Alan Shapiro," Use of the Vorticity Equation in DualDoppler Analysis of the Vertical Velocity Field," Journal of Atmospheric and Oceanic Technology Vol. 19, pp.543-567, 2002.

[7] H.L.Royden, Real Analysis,third edition, Macmillan Publishing Company, New York, 1988.

[8] M. Schult,Spline Analysis, Prentice-Hall, Englewood Cliffs, N.J., 1973.

[9] Alan Shapiro and John Mewes, "New Formulations of Dual-Doppler Wind Analysis," Journal of Atmospheric and Oceanic Technology, Vol. 16, pp.782-792, 1999.

[10] Alan Shapiro," The Use of an Exact Solution of the Navier-Stokes Equations in a Validation Test of a Three-Dimensional Nonhydrostatic Numerical Model," Monthly Weather Review, Vol. 121,pp. 2420-2425.

[11] A. Shapiro, P. Robinson, J. Wurman, and J Gao, "Single-Doppler velocity retrieval with rapid scan radar data." J. Atmos. and Oceanic Technology, 20, pp 1758-1174, 2000.

[12] G.W.Stewart,Introduction to Matrix Computations, Academic Press, New York, 1973.

[13] Roger Temam, Navier-Stokes Equations, North-Holland, New York,1979.

[14] Luther White and Alan Shapiro,"Optimization of wind field retrieval procedures," Applied Mathematics and Computation,171(2005),pp 25-52. 


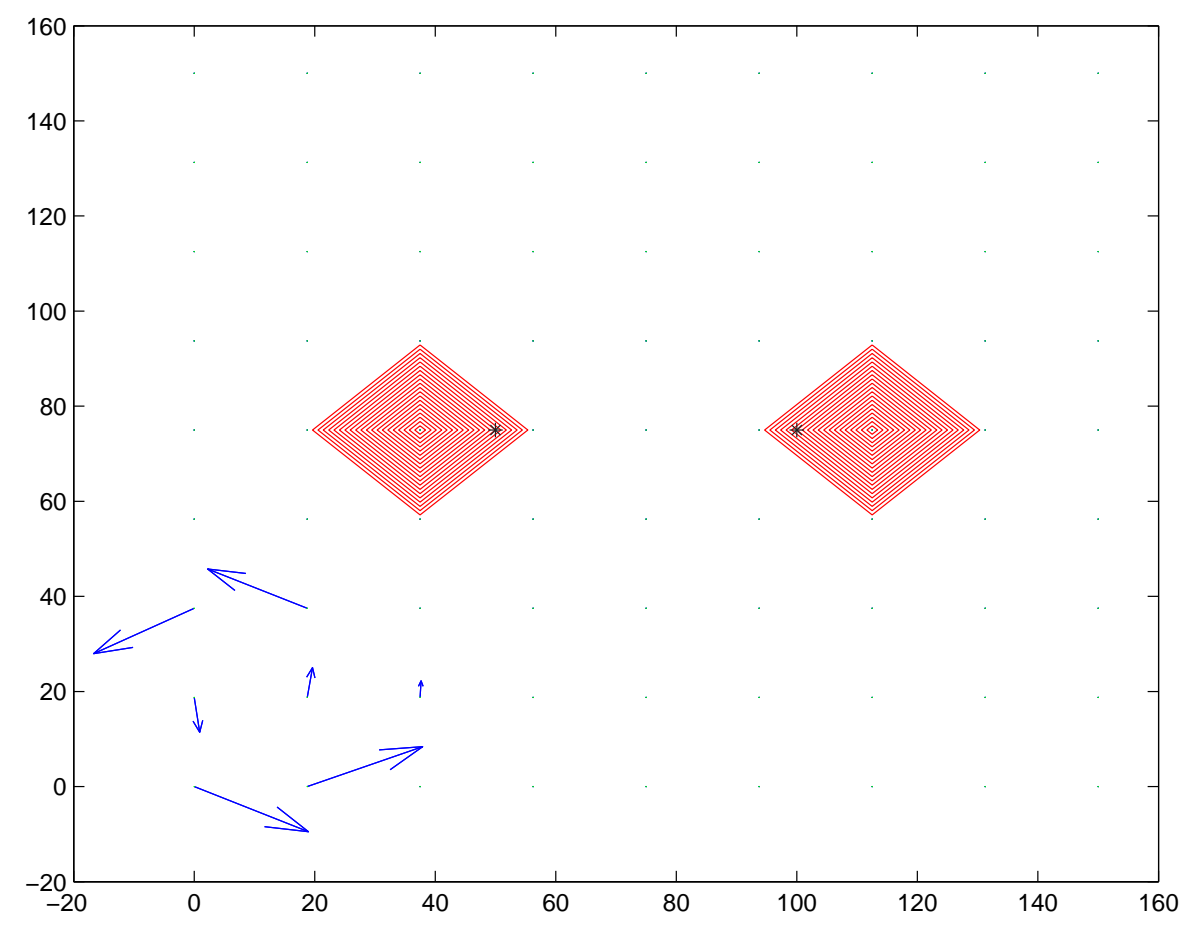

Figure 1: Time $=1$ 


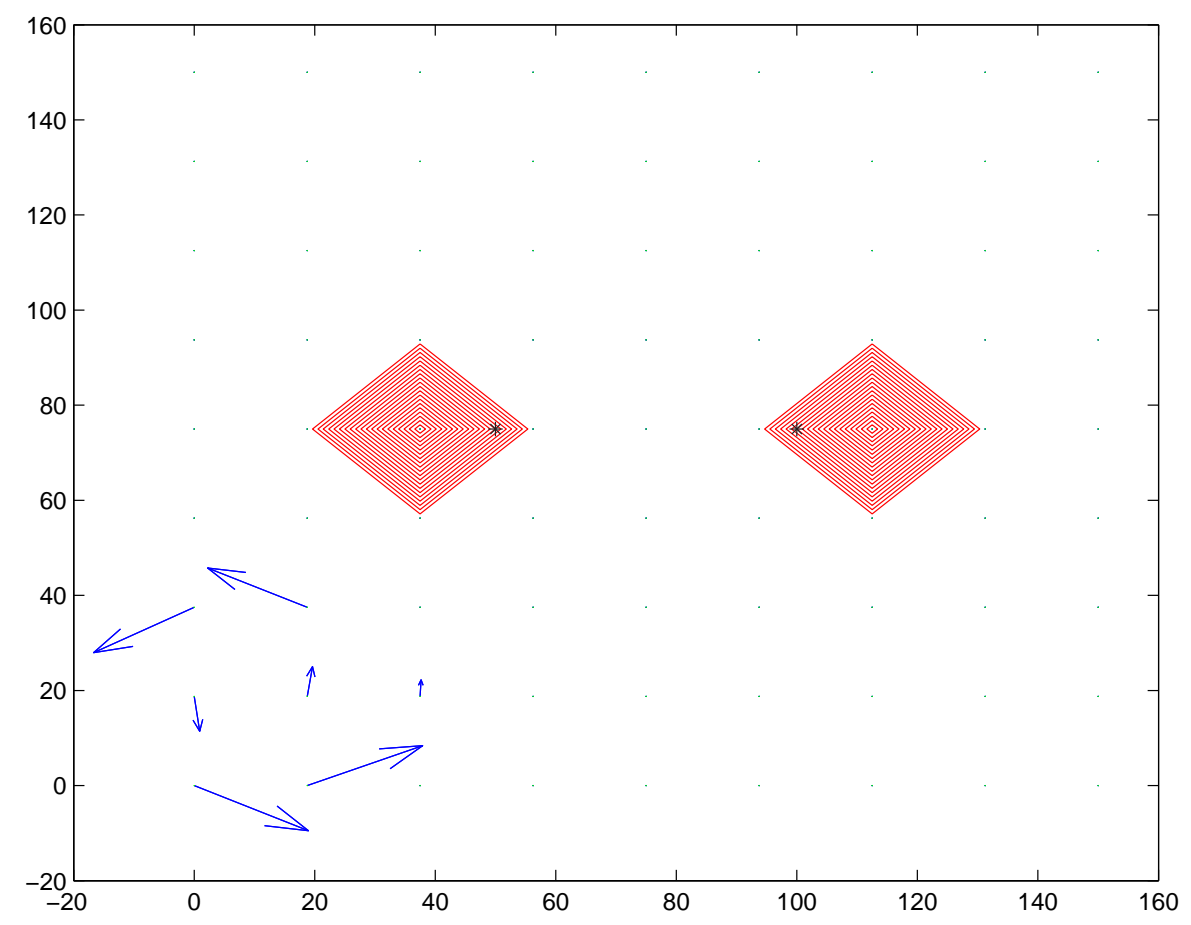

Figure 2: Time $=2$ 


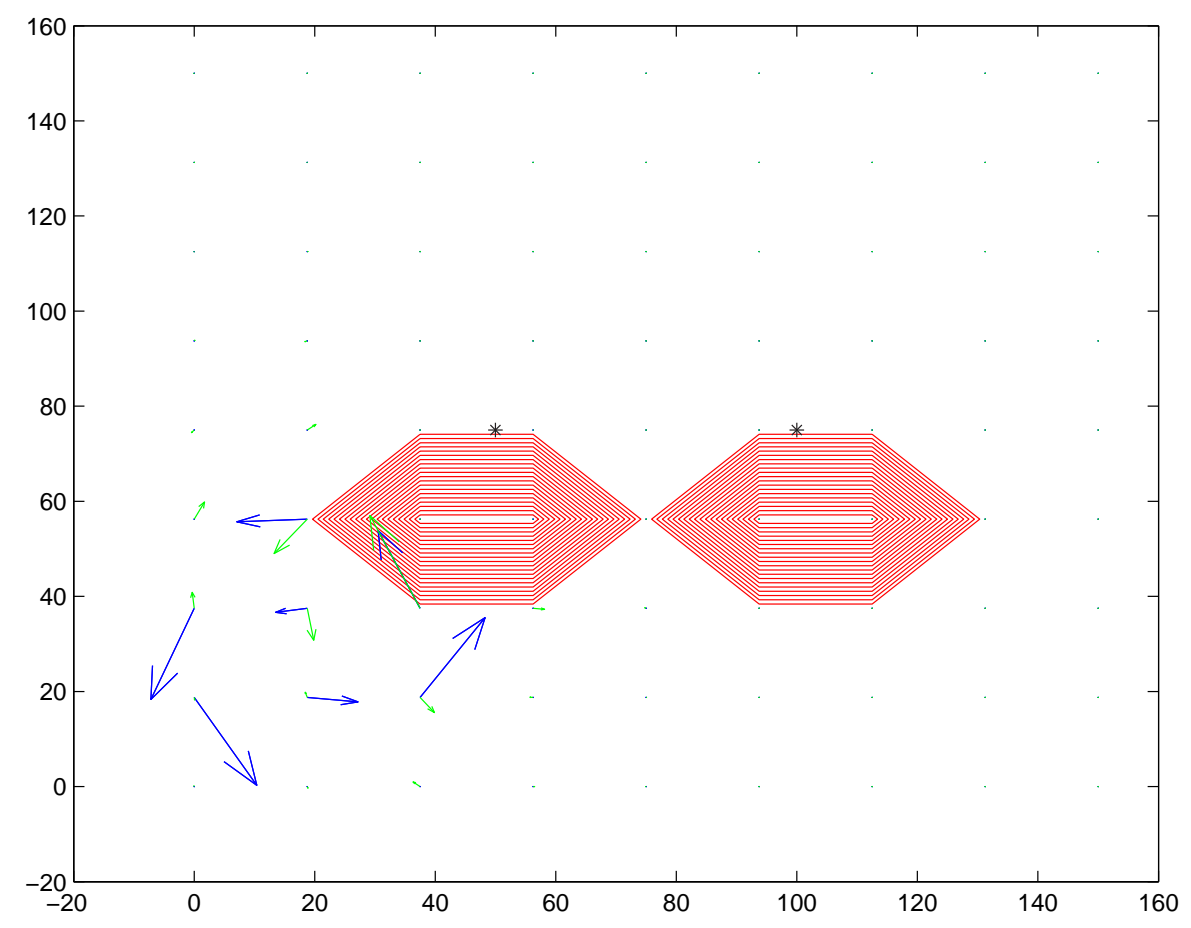

Figure 3: Time $=3$ 




Figure 4 : Time $=4$ 


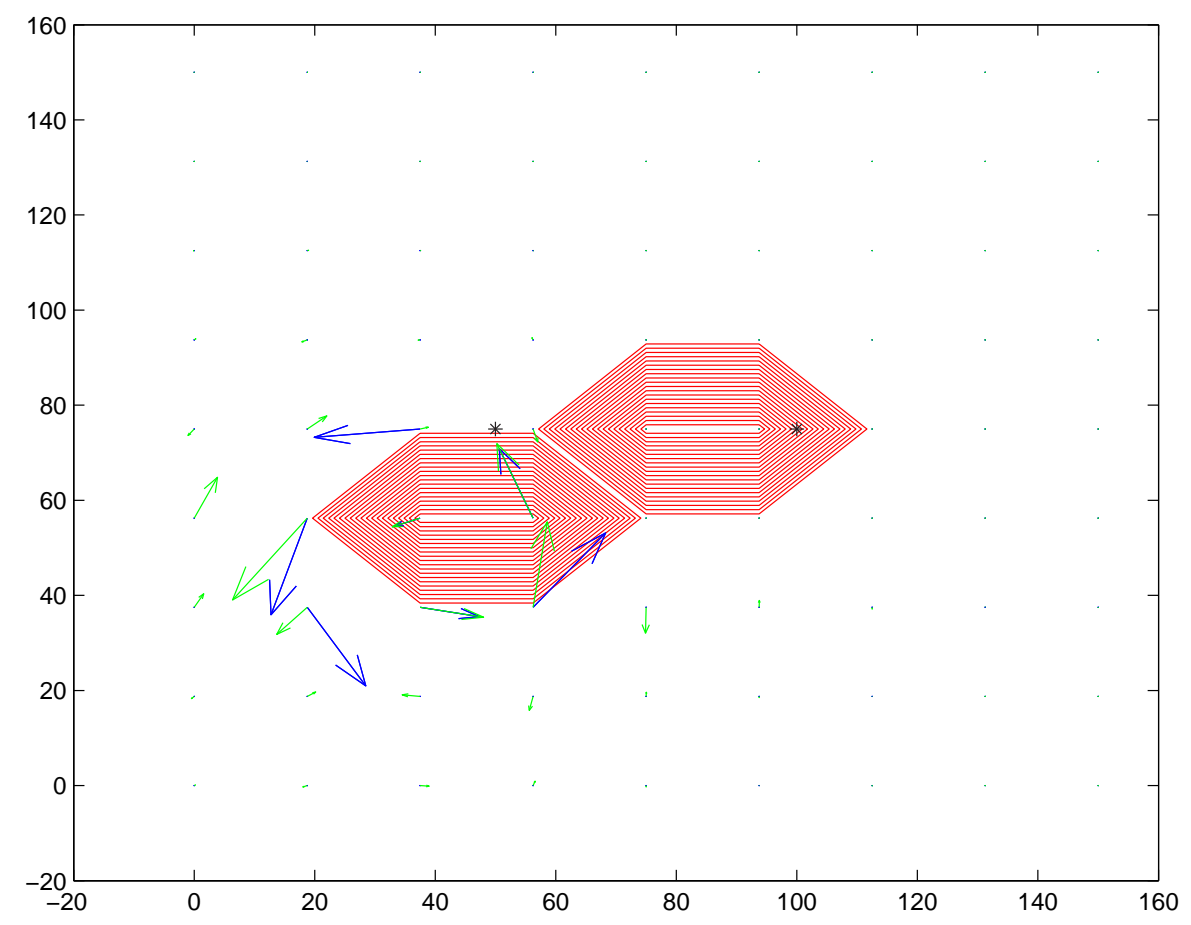

Figure 5: Time $=5$ 


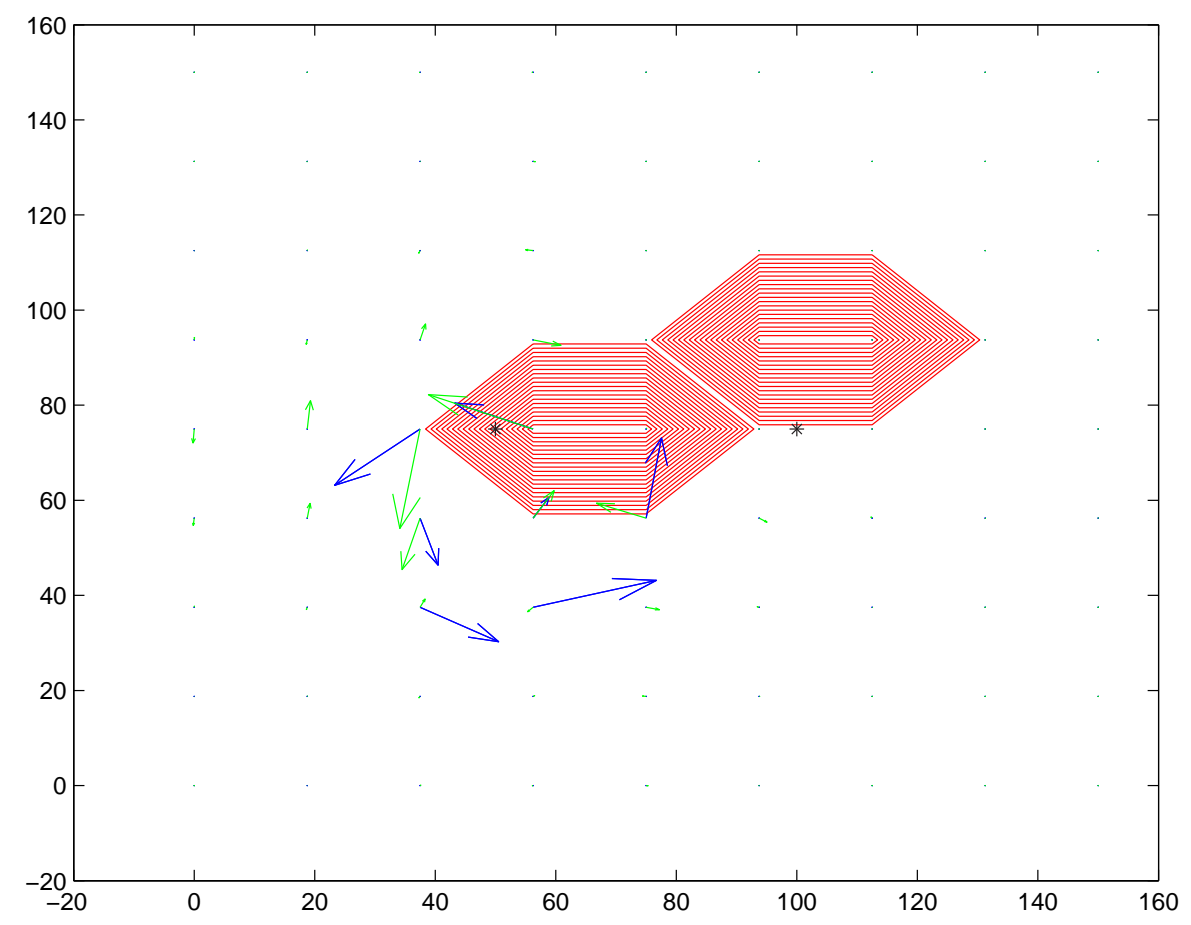

Figure 6: Time $=6$ 


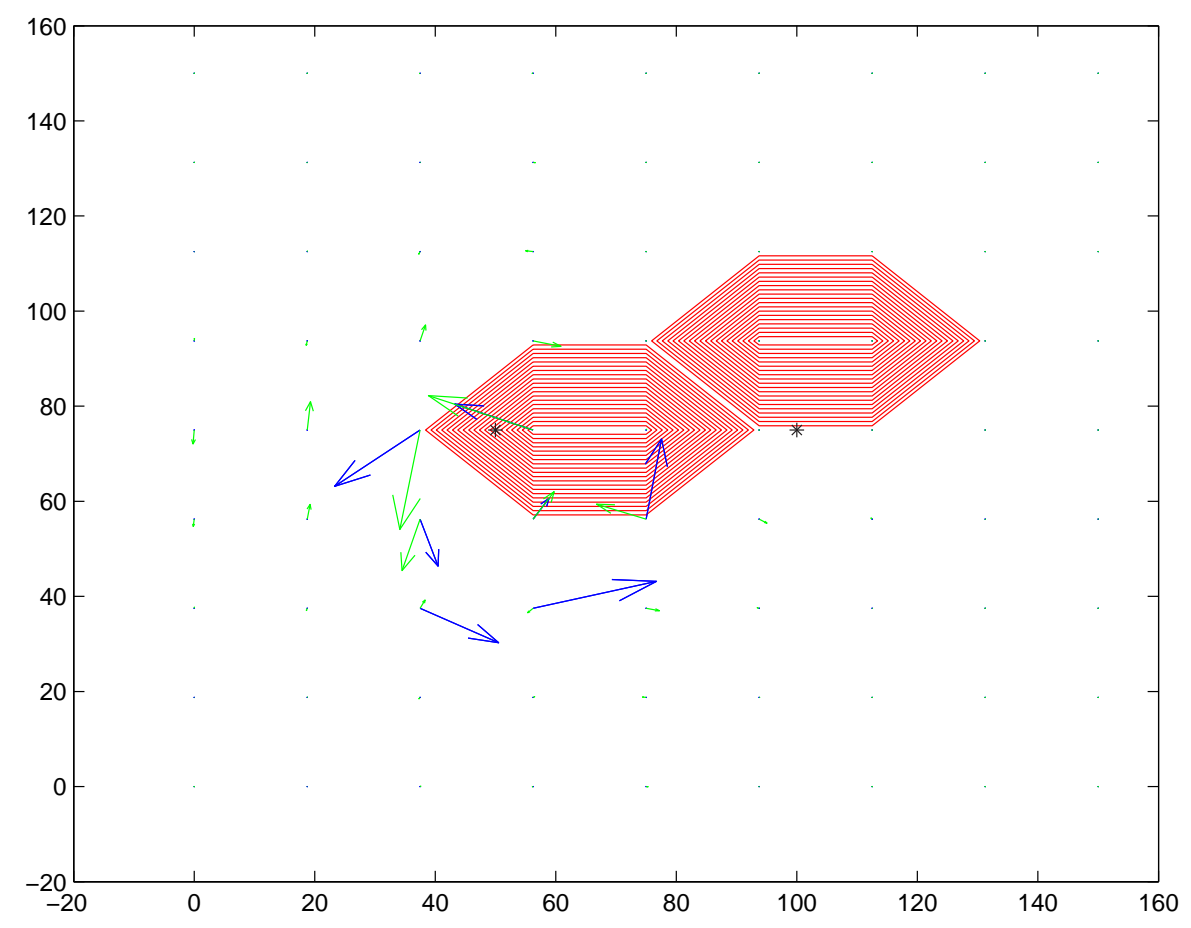

Figure 7: Time $=7$ 




Figure 8: Time $=8$ 


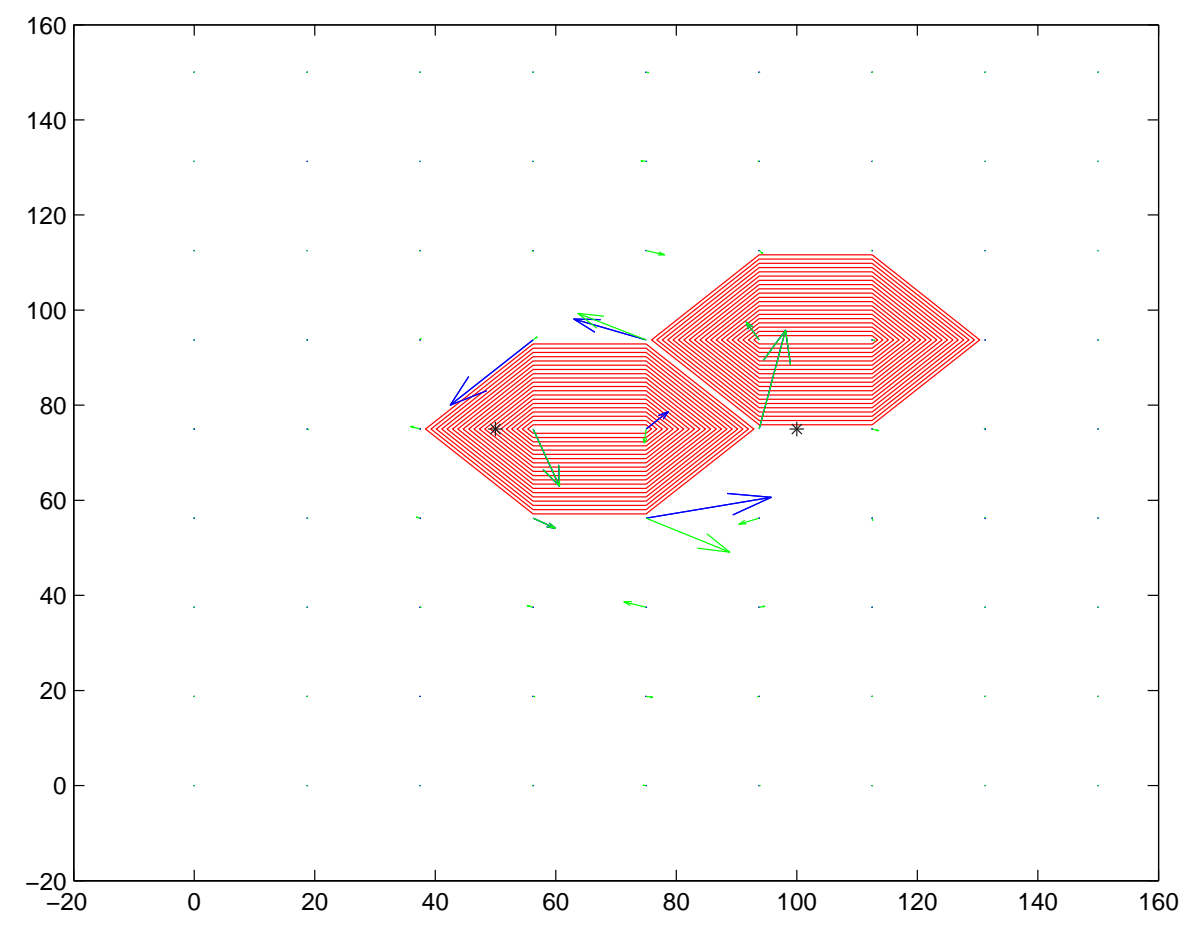

Figure 9: Time $=9$ 


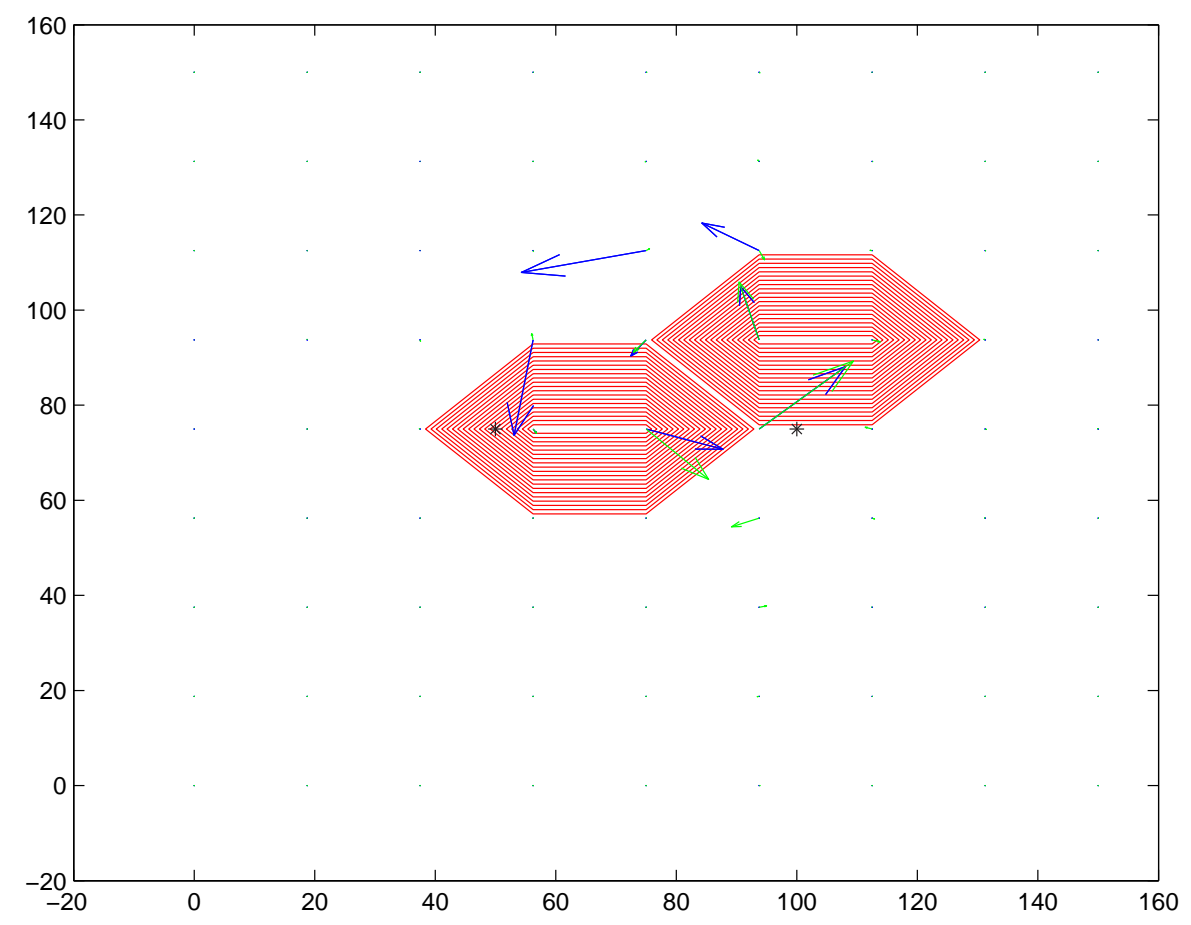

Figure 10: Time $=10$ 


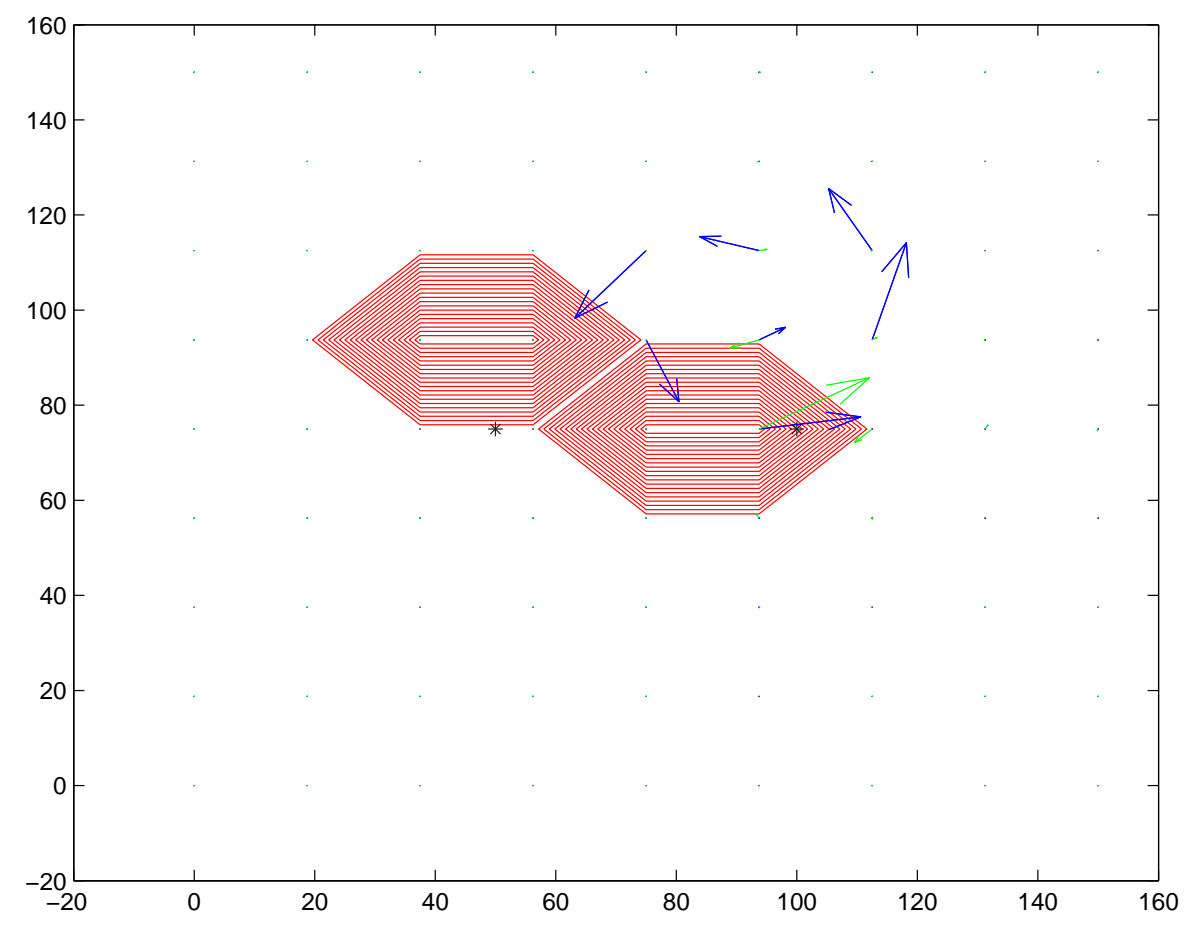

Figure 11: Time $=11$ 


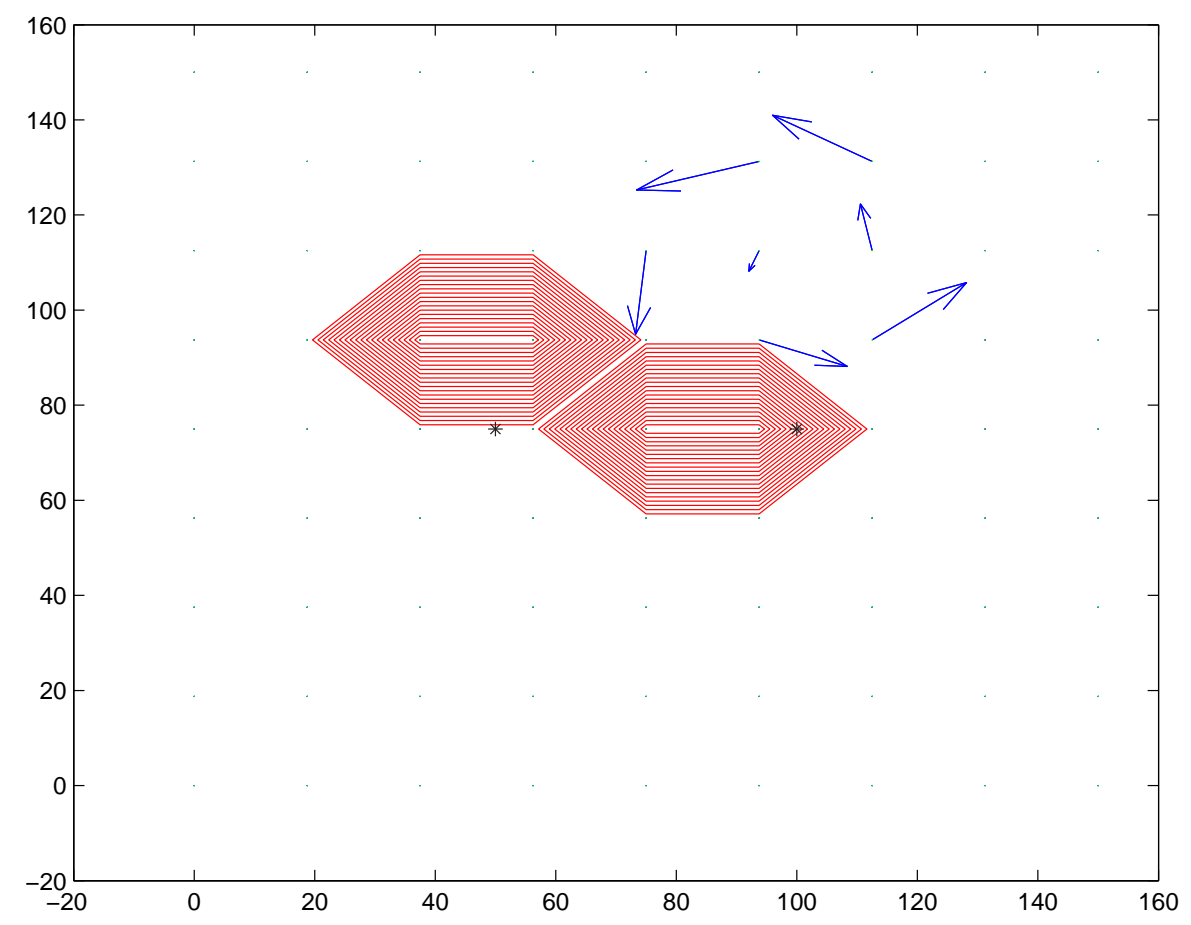

Figure 12: Time $=12$ 


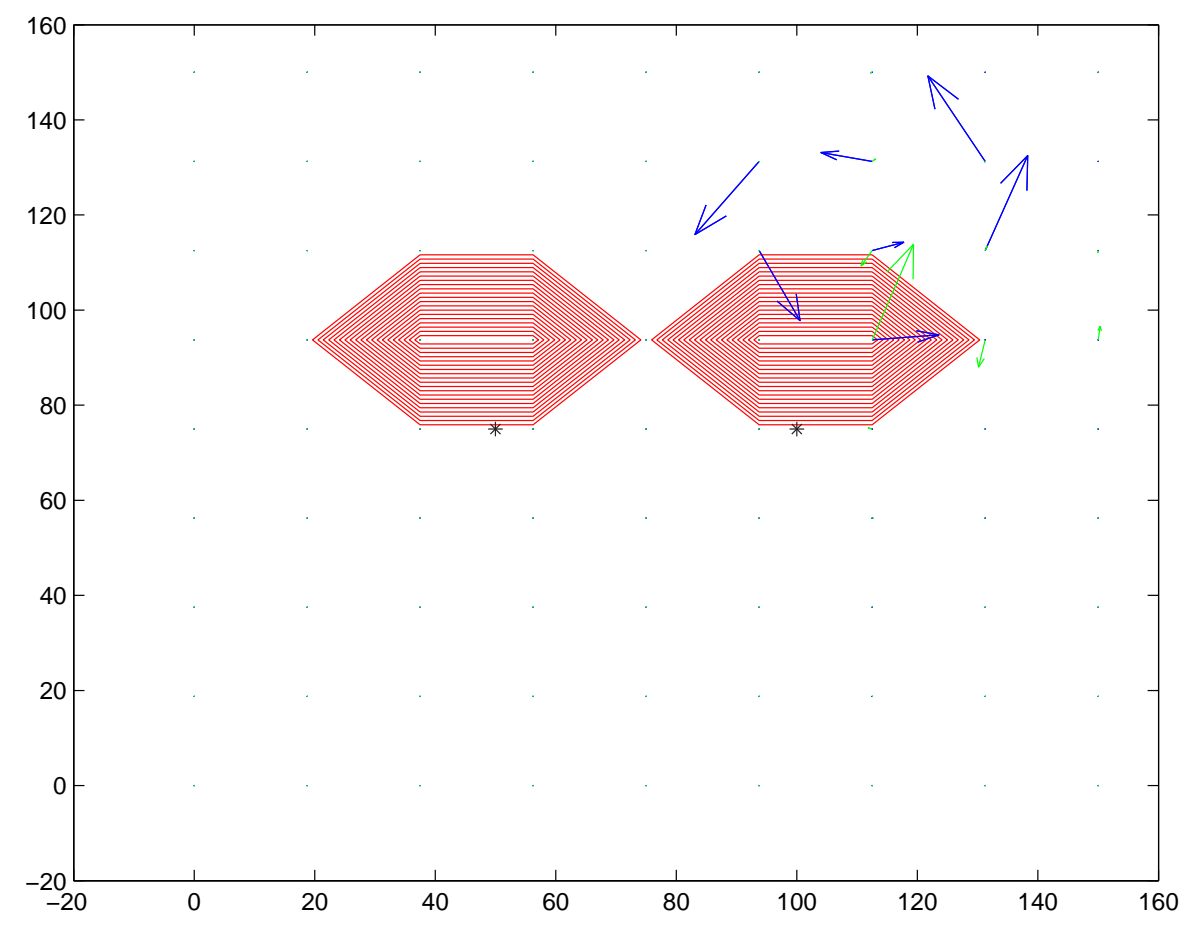

Figure 13: Time $=13$ 


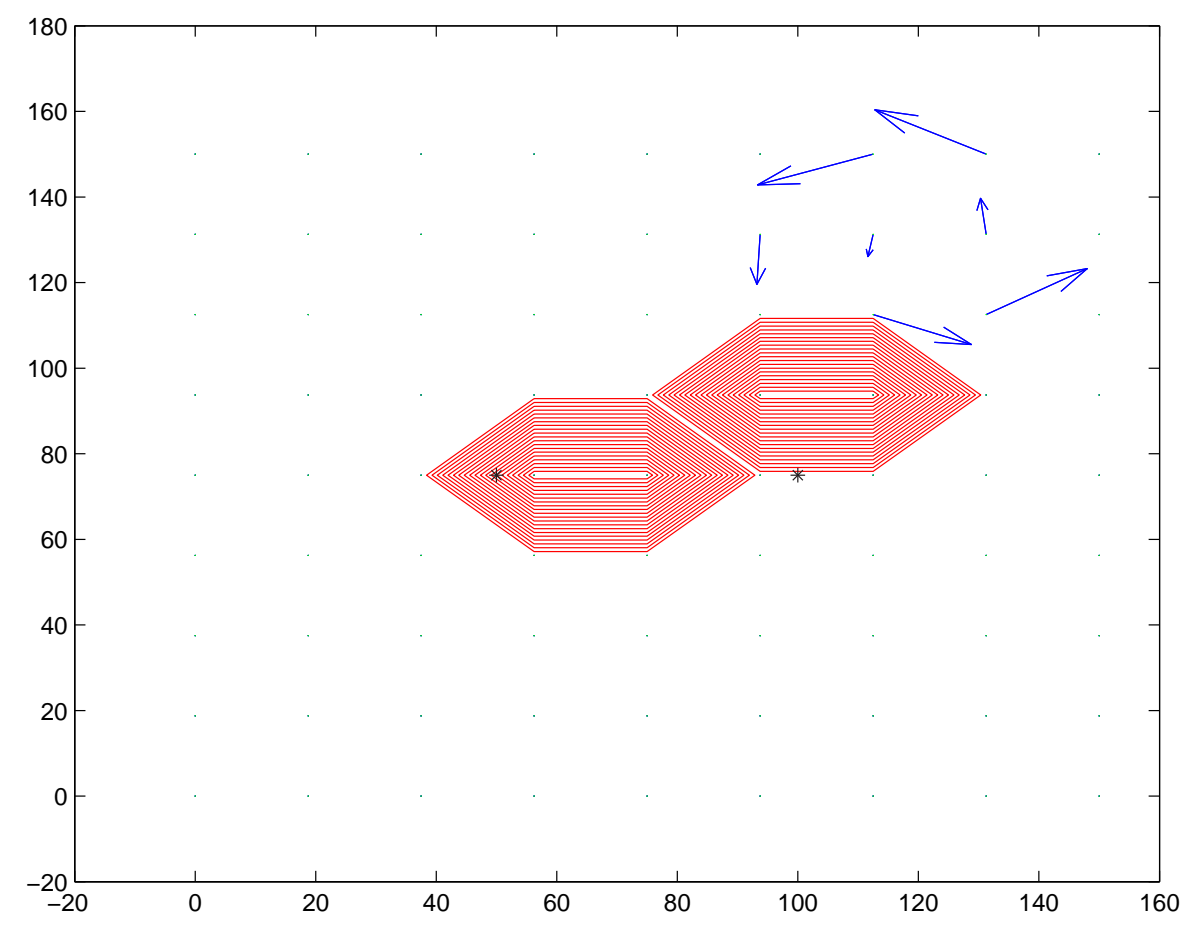

Figure 14: Time $=14$ 


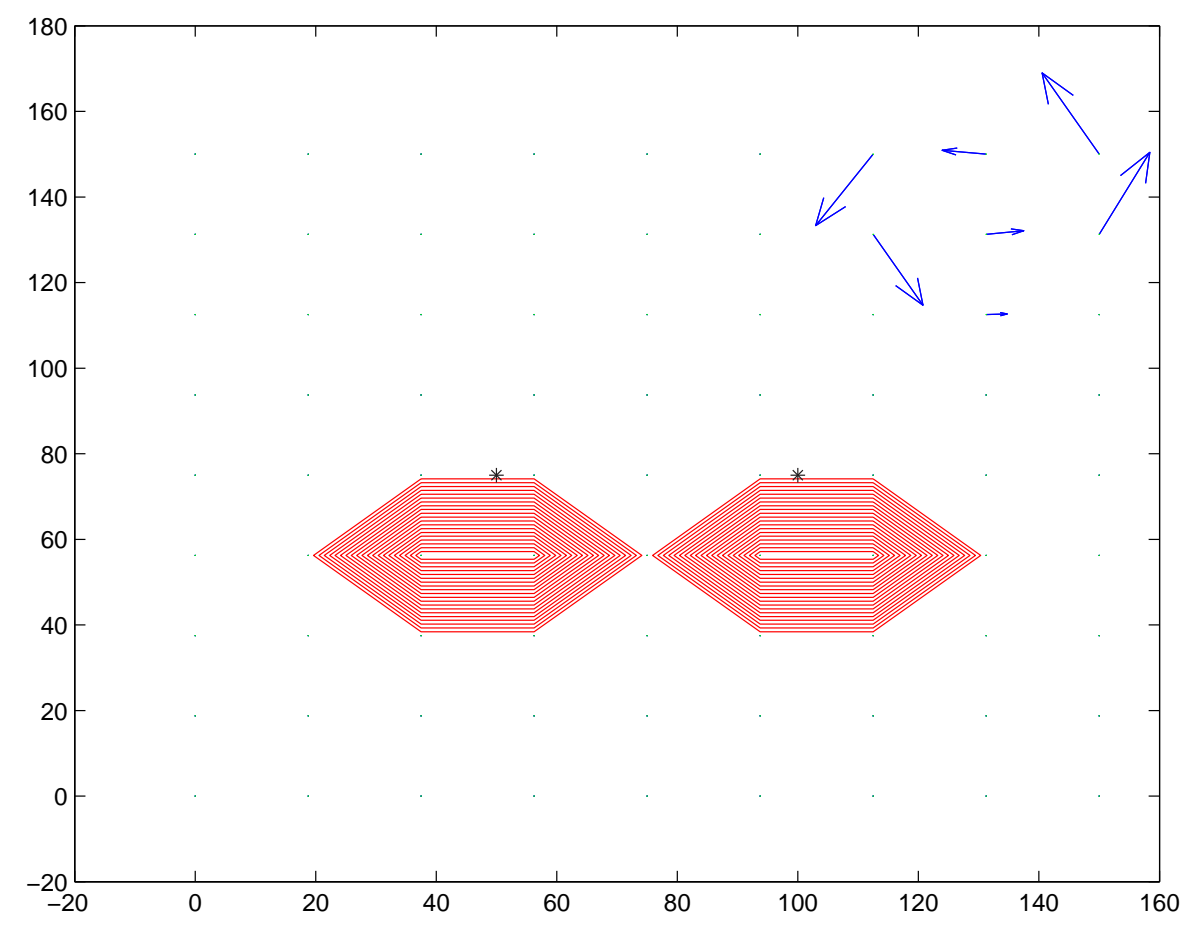

Figure 15: Time $=15$ 